\title{
DETECTION OF THERMAL WATER VAPOR EMISSION FROM W HYDRAE
}

\author{
DAVID A. NEUFELD AND WESLEY CHEN \\ Dept. of Physics 8 Astronomy, Johns Hopkins University \\ 3400 North Charles Street, Baltimore, MD 21218, USA \\ GARY J. MELNICK \\ Harvard-Smithsonian Center for Astrophysics \\ 60 Garden Street, Cambridge, MA 02138, USA \\ THIJS DE GRAAUW \\ SRON Laboratory for Space Research, P.O. Box 800 \\ 9700 AV Groningen, The Netherlands \\ HELMUT FEUCHTGRUBER, LEO HASER AND DIETER LUTZ \\ Max Planck Inst. für Extraterrestrische Physik \\ D-85740 Garching, Germany \\ AND \\ MARTIN HARWIT \\ 511 H Street, SW, Washington, DC, USA
}

\begin{abstract}
We have detected four far-infrared emission lines of water vapor toward the evolved star W Hydrae, using the Short Wavelength Spectrometer (SWS) of the Infrared Space Observatory (ISO). This is the first detection of thermal water vapor emission from a circumstellar outflow.
\end{abstract}

\section{Observations}

Theoretical models of circumstellar outflows (e.g. Goldreich \& Scoville 1976; Chen \& Neufeld 1995) predict that far-infrared water lines will dominate the cooling of the outflowing gas from oxygen-rich evolved stars. As a test of this prediction, we have searched for water emission toward the oxygenrich evolved star W Hydrae. In observations (Neufeld et al. 1996) carried out during the Performance Verification phase of the ISO mission, we detected four far-infrared rotational emission lines of water vapor and one 


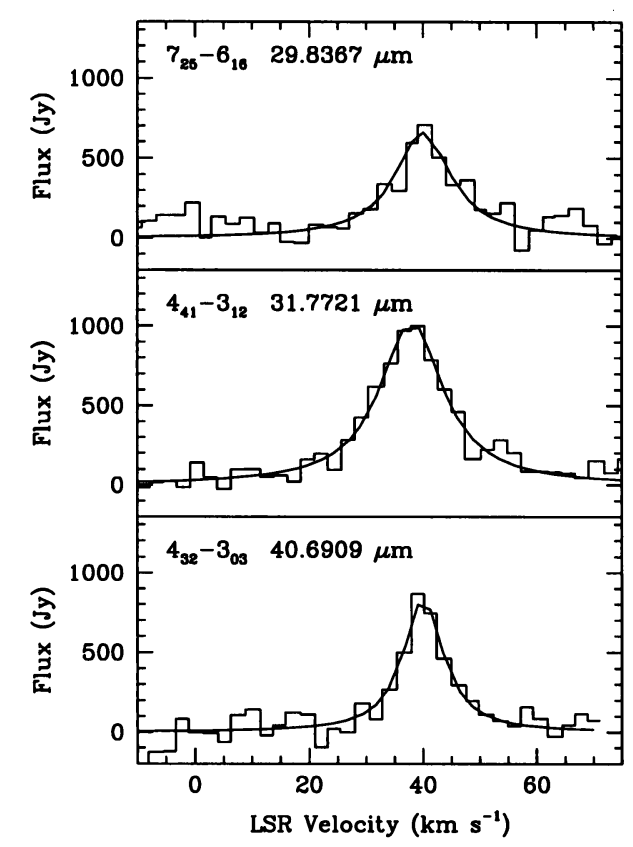

Figure 1. Continuum-subtracted Fabry-Perot spectra from Neufeld et al. (1996) showing the $7_{25}-6_{16}$ line at $29.8367 \mu \mathrm{m}$, the $4_{41}-3_{12}$ line at $31.7721 \mu \mathrm{m}$, and the $4_{32}-3_{03}$ line at $40.6909 \mu \mathrm{m}$.

water absorption feature toward W Hydrae, using the SWS (de Graauw et al. 1996) of $\mathrm{ISO}^{1}$ (Kessler et al. 1996).

Three of the emission lines were observed in the instrument's FabryPerot mode (Figure 1) at a resolving power $\lambda / \Delta \lambda$ of approximately 30000 : the $7_{25}-6_{16}$ line at $29.84 \mu \mathrm{m}$, the $4_{41}-3_{12}$ line at $31.77 \mu \mathrm{m}$, and the $4_{32}-3_{03}$ line at $40.69 \mu \mathrm{m}$. One additional emission line, the $4_{41}-4_{14}$ line at $37.98 \mu \mathrm{m}$, and one absorption feature at $38.08 \mu \mathrm{m}$ that we attribute to a blend of the $13_{13,0}-13_{12,1}$ and the $13_{13,1}-13_{12,2}$ water lines were observed in grating mode (Figure 2) at a resolving power of about 2000 . The observed emission line fluxes are $3.2 \times 10^{-19}, 6.3 \times 10^{-19}, 2.3 \times 10^{-19}$ and $2.8 \times 10^{-19} \mathrm{~W} \mathrm{~cm}^{-2}$ respectively, for the $29.84,31.77,40.69$, and 37.98 $\mu \mathrm{m}$ lines.

\footnotetext{
${ }^{1}$ The Infrared Space Observatory is an ESA project with instruments funded by ESA Member States (especially the PI countries: France, Germany, the Netherlands and the United Kingdom) and with the participation of ISAS and NASA.
} 


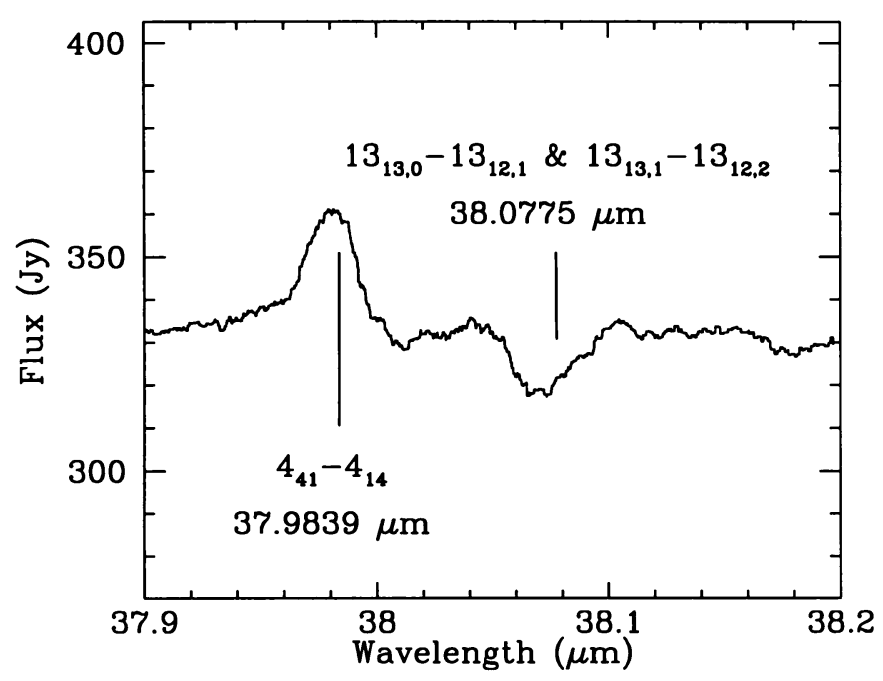

Figure 2. Grating spectrum from Neufeld et al. (1996) showing the $4_{41}-4_{14}$ emission line at $37.9839 \mu \mathrm{m}$ and a water absorption feature at $38.0775 \mu \mathrm{m}$. The wavelength scale is in the rest frame of $\mathrm{W}$ Hya.

\section{Interpretation}

Details of the comparison between observation and theory have been described by Neufeld et al. (1996). To within the possible errors in the flux calibration, the observed emission line fluxes can be accounted for simultaneously by a model similar to that of Chen \& Neufeld (1995), given a mass-loss rate in the range $(0.5-3) \times 10^{-5} \mathrm{M}_{\odot} \mathrm{yr}^{-1}$.

The water emission line fluxes that we have observed toward W Hydrae imply a prodigious luminosity of far-infrared line emission from water. The total flux in all far-IR water emission lines is predicted to be $\sim 10^{-16} \mathrm{~W} \mathrm{~cm}^{-2}$, corresponding to a total luminosity of $\sim 0.3 \mathrm{~L}_{\odot}$. Water emission lines are expected to account for $\sim 0.4,1.5$ and $3 \%$, respectively, of the total 25, 60 and $100 \mu \mathrm{m}$ IRAS band fluxes.

\section{Acknowledgements}

The work of D.A.N. and W.C. was supported by NASA grants NAGW-3147 and NAGW-3183, and that of M.H. by NASA grant NAGW-1261. 


\section{References}

de Graauw, Th., et al. 1996, A\&A, 315, L49

Chen, W., Neufeld, D.A. 1995, ApJ, 452, L99

Goldreich, P., Scoville, N. 1976, ApJ, 205, 144

Kessler, M., et al. 1996, A\&A, 315, L27

Neufeld, D.A., Chen, W., Melnick, G.J., de Graauw, T., Feuchtgruber, H., Haser, L., Lutz, D. 1996, A\&A, 315, L237

\section{Discussion}

Scappini: Are the water emission lines that you have observed in the far-infrared masing or not? Have you checked this point with statistical equilibrium calculations?

Neufeld: We do not expect any of these far-infrared water lines to be masing, given the results of our statistical equilibrium calculations. Nor is there any observational evidence for maser action.

Millar: Could you indicate why mass-loss rates derived from CO show such a discrepancy from that calculated by your model? The derivation of mass-loss rates from $\mathrm{CO}$ observations is a widespread technique.

Neufeld: Mass-loss rates have typically been derived from CO observations using the excitation model of Knapp \& Morris. That model has two shortcomings that might lead it to underestimate the mass-loss rate. Firstly, the work of Mamon, Glassgold \& Huggins has suggested that the CO photodissociation radius is rather smaller than that assumed by Knapp \& Morris; and secondly, the Knapp \& Morris model assumed a temperature profile in the outflowing gas that is probably too high.

Mellema: If the water and CO emissions come from different regions around the star, they could be tracing different episodes of mass loss, if mass loss is time dependent. This could possibly resolve the discrepancy between the derived mass loss rates.

Neufeld: That's a very good point. Because the critical density for the water lines is much higher than that for low- $J$ CO lines, the water-emitting region lies much closer to the star.

d'Hendecourt: What happens to this $\mathrm{H}_{2} \mathrm{O}$ ? Does it form ice grains in the wind? Neufeld: I believe that the dust temperature is too high and the outflow timescale is too short to permit the formation of icy mantles. The water is presumably dissociated by the interstellar UV field in the outer parts of the outflow, giving rise to a shell of $\mathrm{OH}$.

Keene: You haven't yet told us what the water abundance is. Could you tell us what this abundance is relative to $\mathrm{CO}$ and whether it is consistent with what is known about the $\mathrm{C} / \mathrm{O}$ ratio in this star?

Neufeld: We haven't yet investigated the dependence of the predicted line fluxes upon the assumed water abundance. All I can say at present is that the results are consistent with our assumption that water accounts for all the oxygen nuclei that are not bound in $\mathrm{CO}$, but I cannot yet place limits upon the range of assumed water abundances that might provide acceptable agreement with the observed line fluxes. 